\title{
Simulation of Border Control in an Ongoing Web-Based Experiment for Estimating Morphing Detection Performance of Humans
}

\author{
Andrey Makrushin \\ Otto von Guericke University \\ Magdeburg, Germany \\ andrey.makrushin@ovgu.de
}

\author{
Dennis Siegel \\ Otto von Guericke University \\ Magdeburg, Germany \\ dennis.siegel@st.ovgu.de
}

\author{
Jana Dittmann \\ Otto von Guericke University \\ Magdeburg, Germany \\ jana.dittmann@ovgu.de
}

\begin{abstract}
A morphed face image injected into an identity document destroys the unique link between a person and a document meaning that such a multi-identity document may be successfully used by several persons for face-recognition-based identity verification. A morphed face in an electronic machine readable travel document may allow a wanted criminal to illicitly cross a border. This paper describes an improvement of our ongoing web-based experiment for a border control simulation in which human examiners should first detect high-resolution morphed face images and second match potentially morphed document images against "live" faces of travelers. The error rates of humans in both parts of the experiment are compared with those of automated morphing detectors and face recognition systems. This experiment improves understanding the capabilities and limits of humans in withstanding the face morphing attack as well as the factors influencing their performance.
\end{abstract}

\section{KEYWORDS}

Face morphing attack, $\mathrm{ABC}, \mathrm{MAD}$, Human experiment

\section{ACM Reference format:}

Andrey Makrushin, Dennis Siegel and Jana Dittmann. 2020. Simulation of Border Control in an Ongoing Web-Based Experiment for Estimating Morphing Detection Performance of Humans. In IH\&MMSec '20: 8th ACM Workshop on Information Hiding and Multimedia Security, fune 22--24, 2020, Denver, CO, USA, 6 pages. https://doi.org/10.1145/10.1145/3369412. 3395073

\section{Introduction}

Face recognition is a widely accepted and rapidly developed technology that has currently been involved in many spheres of our life in which biometric user authentication is required. One example is an automated border control (ABC) based on electronic machine readable travel documents (MRTD). Back in 2002, face has been selected by the International Civil Aviation Organization (ICAO) to be the primary biometric modality used in MRTD. In recent years, the number of $A B C$ systems in airports making use of automated face recognition (AFR) has

\section{(ब) $\odot \Theta$}

This work is licensed under a Creative Commons Attribution-NonCommercialNoDerivs International 4.0 License.

IH\&MMSec '20, fune 22-24, 2020, Denver, CO, USA

(C) 2020 Copyright is held by the owner/author(s).

ACM ISBN 978-1-4503-7050-9/20/06.

https://doi.org/10.1145/3369412.3395073 rapidly increased because it speeds up traveler's identity verification and avoids queues. Another reason is that humans are prone to errors in matching unfamiliar faces [1] and passport officers are no exception [2]. Although face verification has many advantages, it is vulnerable to presentation [3] and morphing attacks [4]. Here, we examine the human ability to counter morphing attacks and compare it to the performance of algorithms.

Technically, a face morphing attack is comprised of two steps and has two actors: an attacker (Mallory) and an accomplice (Alice). Alice applies for a new identity document with a morphed face image of her and Mallory's faces. If an officer accepted the image, the genuine document would be issued. Mallory uses this fraudulently obtained genuine (FOG) document for identity verification claiming to be Alice. The vulnerability exploited by the attack is a possibility to apply for a new document with an off-site produced photograph. If a morphed photo biometrically resembles all constituent faces and also contains no visual artifacts, it is highly likely that a passport officer accepts the photo to be used in a document and that commonly used AFR systems successfully match the photo against any of the constituent faces [5][6]. Face morphing has never been as easy and popular as at the moment. There exist many commercial and free software tools enabling the creation of morphed faces in little time with a little assistance. Hence, even inexperienced forgers are able to create high-quality face morphs. Moreover, high-quality morphs can be created fully automatically [7].

Countering morphing attacks is possible by administrative or technical means. On the one hand, prohibiting an off-site photograph production would limit the problem, but should be seen as a measure of last resort. On the other hand, technical solutions like training of passport and border officers or deployment of dedicated morphing attack detection (MAD) approaches are possible. However, previous studies do not give a clear answer whether training of humans would strongly decrease their error rates, and who performs better humans or algorithms. In this study we endeavor to answer the following research questions:

- Is the performance of skilled and unskilled human examiners significantly different?

- Which samples push participants to their limits?

- Is there a correlation between decision time and error rates?

- Do algorithms perform better than humans on the same samples?

- Do the results from the new experiment contradict or confirm the results of previous experiments? 
Our contribution can be summarized as follows: we point out the deficiencies of the existing ongoing web-based experiment [8] and launch a new experiment in which we first let participants perform morphing detection without a reference image and compare the results with those of two exemplarily selected MAD approaches. Second, we let people perform biometric matching of a potentially morphed passport photograph against a "live" face and compare the results with those of two exemplarily selected AFR systems. The new experiment has a higher diversity of models, includes an option to reject a verification trial because of a morphed passport photograph, includes printed and scanned document images, better balances numbers of images in different parts of the experiment, has an improved video presentation, and includes a more thoughtful questionnaire. The evaluation is done based on answers of 189 participants.

Hereafter, in Section 2, we discuss the existing experiments on morphing detection performance of humans. In Section 3, we describe our experiment in detail. In Section 4, we evaluate the results and compare error rates of humans and algorithms. Section 5 concludes the paper with the summary of results.

\section{Related works}

Border control is comprised of two procedures: an authenticity and integrity check of a travel document and biometric authentication of a traveler based on the biometric record stored in the document. Since the face morphing attack implies usage of FOG documents, the authenticity and integrity checks are irrelevant. An exposure of an illicit border crossing could be done only as a part of biometric authentication which is carried out either by border guards or ABC systems. A border guard compares a printed passport photograph with a traveler's face while an $\mathrm{ABC}$ system compares a digital passport photograph with a "live" face image making use of an integrated AFR system. For comparison of face recognition performances of humans and AFR systems see [9].

The first study on comparison of humans and AFR systems to counter the face morphing attack is conducted in [5]. 80 morphs were manually generated and 44 border guards and 543 laymen were asked to match these morphs against original face images. Border guards accepted $91.67 \%$ genuine and $74.92 \%$ morphing trials, while laymen $87.76 \%$ genuine and $57.55 \%$ morphing trials indicating that border guards are not better than laymen in this task. Morphing acceptance rates (MAR) of three commercial AFR systems with the same images were unacceptably high. High MAR of two AFR systems were also confirmed in [6]. In [8], it is noticed that such a high MAR is rather pessimistic and may be caused by high similarity between face images used for morphing and for identity verification.

Human performance in matching "live" faces against potentially morphed passport images is addressed in [10]. The participants were first unaware of morphing leading to $68 \%$ MAR. Then a rudimentary morphing detection guidance was provided to participants and the third option was included - "reject verification trial because of a morphed passport photograph". MAR dropped to $21 \%$ indicating that awareness of potential morphing highly reduces the probability to accept morphing trials. An AFR system on a mobile phone demonstrated $27 \%$ MAR with the same morphs. In [11], the same authors once again investigated how much human error rates drop after coaching and tried to establish the relationship between mismatch accuracy on an unfamiliar face matching test and morph detection ability. They concluded that providing passport officials with morph detection training can significantly decrease acceptance of morphed face images and also noticed that individuals who better recognize different people on similarly looking images may also better detect morphed faces.

In [12], the aforementioned experiment was replicated with facial morphs of higher quality. It was inferred that training does not really improve the morphing detection performance. The authors also conducted experiments on morphing detection without a reference face image, simulation of a border control scenario, where travelers present their passports and talks to officers, and officers compare passport images with "live" faces. In the end, the same morphs were examined with an algorithm performing a morphing detection without a reference and concluded that computer-aided morphing detection may be a better solution. The reason for contradicting results in [11] and [12] is the different quality of morphs. Low-quality morphs in [11] have artifacts evident to the naked eye while high-quality morphs in [12] do not. A trivial tutorial demonstrating the detection clues would improve the performance of only unskilled examiners and only with low-quality morphs. High-quality morphs have no visible artifacts, making any training obsolete.

An experiment on morphing detection without a reference image performed by humans and by an MAD approach was also conducted in [7]. The authors claimed that their morphs are visually faultless and reported $44.60 \%$ MAR with $43.64 \%$ false rejection rate (FRR) of humans. The MAD approach yielded $12.11 \%$ and $12.97 \%$ MAR for two different types of morphs.

Our first ongoing web-based experiment simulating border control was introduced in [8]. The performances of skilled and unskilled human examiners in morphing detection/matching were compared to each other and to those of algorithms. The average MAR in the detection scenario (referred to as FNR) yielded $15.41 \%$ and in the matching scenario $34.65 \%$. In contrast, the best algorithms with properly adjusted decision thresholds perfectly distinguished genuine and morphing trials in both experiments. The authors concluded that biometric matching is significantly more prone to errors than morphing detection, skilled participants perform on average only slightly better (within 3\%) than unskilled, algorithms demonstrate lower error rates than humans in both tasks, and the detection rate strongly depends on the quality of a morphed image. Here, we revise our experiment in [8] aiming at reducing its biases and deficiencies. For instance, all models were of European descent, or no distinction in the matching experiment can be done regarding whether the trial was rejected because of a morphed passport photograph or because two different subjects were presented. 


\section{Our experiment}

Our experiment is an ongoing web-based questionnaire that is available at: https://bit.ly/2M6oA2C. The participants are free to use a smart-phone, a tablet or even a regular computer to complete it. The available languages are English and German.

There are two kinds of subjects taking part in the experiment: models (image donors) and examiners. Photographs and videos of models are presented to examiners while examiners play a role of border guards deciding whether models are allowed to pass the border or not. All models provided written informed consent that digital photographs and videos of their faces may be used for scientific research and published in papers. The experiment consists of four parts: 1) briefing of examiners on possible morphing artifacts, 2) morphing detection without a reference image, 3) matching of passport photographs against "live" faces, and 4) a questionnaire to collect metadata of examiners.

\subsection{Motivation and improvements}

The main objective of our experiment is to estimate the likelihood that a human examiner is deceived by an automatically morphed face image in a simulated border crossing environment. Hence, the core of our experiment is the third part - matching of passport photographs against "live" faces (see 3.4). The first two parts (see 3.2 and 3.3) can be seen as a warm-up.

There are only five models presented in [8] and they all are of European descent. Here, the number and diversity of models are dramatically increased. We collected 50 images and 34 videos of 50 models from all racial backgrounds. The number of matching trials increased from 15 to 25 incl. 10 morphing, 5 impostor and 10 genuine trials. The previous experiment does not include impostor trials at all making it impossible to compare matching performances for impostor and morphing trials. Morphs were created using the approach from [13] designated as "combined morphing". No retouching was applied meaning that morphs may have evident visual artifacts. All passport images were printed and scanned to simulate image processing at a passport issuing office. For creating a feeling of working under time pressure, we added a progress bar changing its color from blue to red. Note that the trial is not canceled after the progress bar is full. The video resolution is dramatically increased making the face size in both video and a passport image of the same order of magnitude. For more thorough evaluation, we improved the questionnaire at the end of the experiment. We slightly changed the choices and the scales in the questions: "Which face parts did you mostly focus on?", "How many models are you acquainted with?" and "What is your experience with face morphing?" which should enable us to better understand the decisions of examiners. We also ask whether an examiner has already taken part in our first experiment [8]. Finally, we evaluate the decision time of examiners and check which face regions give more hints for detection.

\subsection{Tutorial on morphing detection}

In order to avoid the situation (as occurred in [10]) that a rudimentary training greatly improves performance of exami- ners who are unaware of morphing, we first provide examiners with a basic knowledge of facial morphing and demonstrate two exemplary morphed face images with typical morphing artifacts (see Figure 1). This coaching does not make an expert out of a layman but helps to avoid random guessing by examiners.

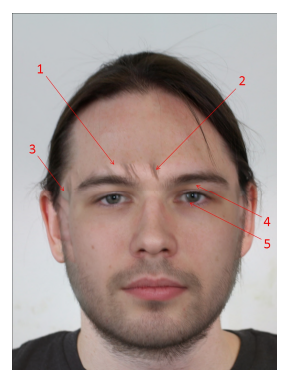

(a)

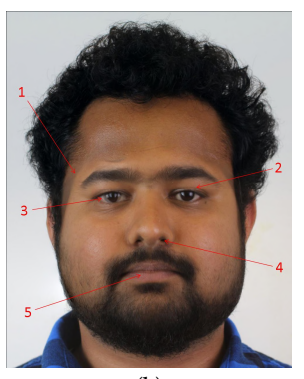

(b)
Figure 1: Typical morphing artifacts: a "swimming cap" effect (a1, b1); ghosting artifacts in the hair (a2), at the temples (a3), underneath the eyebrows (a4); double irises (a5, b3), eyelid folds (b2), nostrils (b4), lip contours (b5).

\subsection{Morphing detection}

The morphing detection part consists of 15 trials: 5 genuine (G1, $\mathrm{G} 2, \ldots, \mathrm{G} 5)$ and 10 morphed face images (M1, M2,..., M10). In each trial, an examiner looks at a passport photograph and decides whether it is morphed or not. Since no further information about the photograph is given such detection is referred to as "blind" or single image detection. In contrast to a real-life, an examiner decides solely based on visual morphing artifacts making no use of a model's metadata. Figure 2 shows two examples. The first one (a) is a morphed female face that was correctly detected by $32.3 \%$ of examiners and the second (b) morphed male face correctly detected by $93.7 \%$. The images are normalized to have a constant width of 1000 pixels and the height varies from 1038 to 1465 pixels. Note that the images can be zoomed in/out on the user's display.

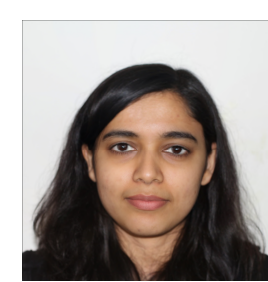

(a)

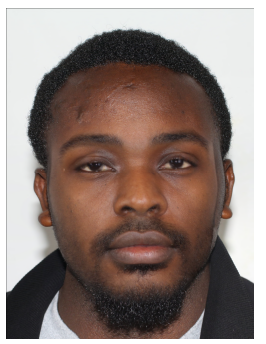

(b)
Figure 2: Morphed face images presented in the detection part of the experiment: (a) challenging sample M2; (b) simple sample M3.

As an alternative to human-based morphing detection, we examine two MAD approaches, one based on localization and counting of keypoints [14], and the other based on Deep Convolutional Neural Networks (DCNN) [15] designated in the original work as "naive". The keypoint-based morphing detector indirectly quantifies the blending effect as an indispensable part of the morphing process. Blending leads to a reduction of face details and therefore to a reduction of "significant corners" and edge pixels. The detector counts the relative number of keypoints in the face region detected by different approaches as well as the 
relative number of edge pixels. For classification, a linear support vector machine was trained based on 24-dim feature vectors with a dataset of 2000 genuine and 2000 morphed high-resolution passport images. Morphs are created using the approaches from [7][13]. The DCNN-based morphing detector is based on the VGG19 network. Transfer learning is applied to build a binary classifier from the classification model originally trained for the ILSVRC challenge. The training dataset is comprised of approximately 1900 genuine images and the same number of morphs. Genuine images were collected from several public face databases and scraped from the internet. For generation of morphs, two approaches from [16] were applied. The training set was augmented by the filtered versions of images applying several standard filters like blurring or adding noise. Prior to feeding into the network, the images were normalized including in-plain rotation, face cropping and scaling to $224 \times 224$ pixels.

\subsection{Face matching}

The face matching part consists of 25 trials: 10 genuine (G1, G2, ..., G10), 5 impostor (I1, I2, ..., I5) and 10 morphing trials (M1, $\mathrm{M} 2, \ldots, \mathrm{M} 10)$. In each trial, an examiner looks at a passport photograph and simultaneously watches the video of a traveler approaching her/him and then making head rotations. The examiner should either accept the traveler for passing the border, or reject the traveler because the passport is from a different person, or reject the traveler because the passport photograph is morphed. Figure 3 shows two trials. The first one (a) was falsely accepted by $74.6 \%$ of examiners and the second (b) by $32.8 \%$. Motivated by the ICAO standard [17], all passport photographs are scaled to 413x532 pixels, saved in JPEG format, printed and scanned. The re-digitalization should simulate the process of a photograph's processing in a passport issuing office. The HD ready videos have a resolution of $1280 \times 720$ pixels.

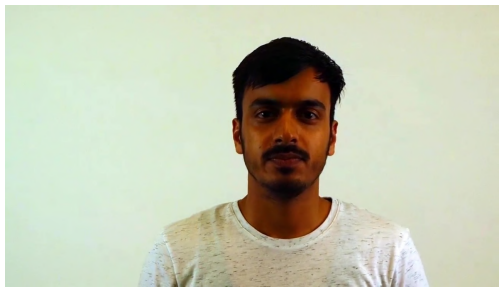

(a)

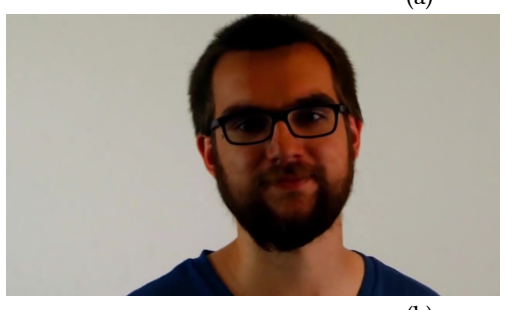

(b)
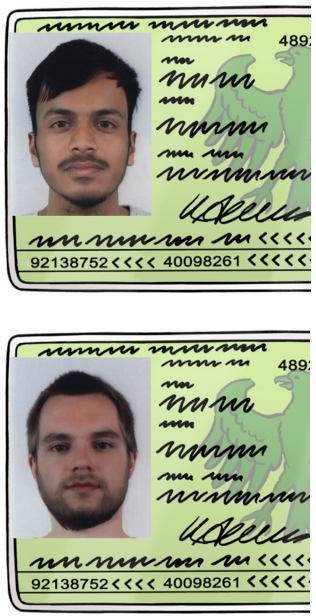

Figure 3: Video snapshots and morphed passport photographs from the matching part of the experiment: (a) challenging sample M1; (b) simple sample M10.

As an alternative to human-based face matching, we examine two AFR systems: Dermalog Face Recognition [18] representing a commercial off-the-shelf (COTS) solution and a face recognition tool provided in the open source programming library Dlib
[19]. The Dermalog Face Recognition is proprietary commercial software with no information disclosed about the face recognition performance and algorithms. The face matcher returns scores from 0 to 100 with higher numbers for higher similarity. The decision threshold recommended for a high-security level is 80. The face recognition tool in the Dlib library was added in the release 19.3 from February 2017 [20]. The slightly modified ResNet-34 network with 29 convolutional layers was trained from scratch based on approx. three million face images collected from diverse open datasets. The classification model is stored in 'dlib_face_recognition_resnet_model_v1.dat'. Input face images are scaled to $150 \times 150$ pixels and mapped by the network to 128 dim feature space in which all identities are supposed to be represented by non-overlapping balls of 0.6 radius. Images of the same identity should be close to each other(low matching scores) while images of different identities far apart (high matching scores). With the distance threshold of 0.6, the model obtains an accuracy of $99.38 \%$ on the Labeled Faces in the Wild benchmark, which was a decent performance back in the beginning of 2017 .

\section{Evaluation}

The experiment was launched on August 7th, 2019. For the evaluation, we took a snapshot on November 27th, 2019, 1 p.m. with 222 participants. Since some participants are acquainted with one or several models and their decisions are biased, we filtered them out and ended up with 189 records. There are 21 participants who claimed to have experience with face morphing (referred to as skilled examiners) and 168 participants who claimed to have no experience (referred to as unskilled examiners).

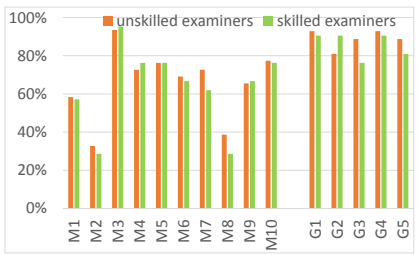

(a)

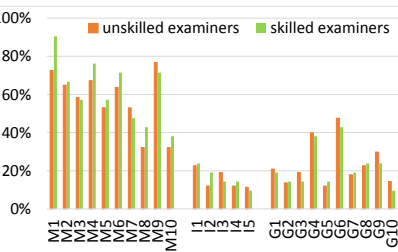

(b)
Figure 4: Error rates of humans: (a) morphing detection with TPR for morphed (M1, M2, ..., M10) and TNR for genuine images (G1, $\mathrm{G} 2, \ldots, \mathrm{G}$ ); (b) matching with MAR for morphing (M1, M2, ...,M10), FAR for impostor (I1,I2,...,I5) and FRR for genuine trials $(\mathrm{G} 1, \mathrm{G} 2, \ldots, \mathrm{G} 10)$

\subsection{Detection experiment}

4.1.1 Performance metrics. Morphing detection is a binary classification problem with morphed images as positive and genuine images as negative samples. The standard metrics are the false positive rate (FPR) and false negative rate (FNR). FPR is the fraction of genuine images falsely classified as morphs (also called false alarms) and FNR is the fraction of morphs falsely classified as genuine images (also called misses). Within the context of presentation attack detection, FPR is denoted as BPCER and FNR as APCER [21]. We also use the opposite rates: the true positive rate (TPR) and true negative rate (TNR) as well as half total error rate (HTER) as an average of FPR and FNR to compare performances in an easier way.

4.1.2 Results of humans. Figure 4a shows morphing detection rates of human examiners. For morphs, the average TPR is 
$65.40 \%$. Surprisingly, unskilled examiners perform better than skilled (average TPR $65.65 \%$ vs. $63.33 \%$ ). There is a negative correlation between the morph quality and the TPR e.g. the TPR with the "simple" morph M3 in Figure $2 \mathrm{~b}$ is $93.45 \% / 95.24 \%$ for unskilled/skilled examiners while the TPR with the "challenging" morph M2 in Figure 2a is $32.74 \% / 28.57 \%$ for unskilled/skilled examiners. Note that for M2, the TPR of skilled examiners is even lower than of unskilled. It indicates that after rudimentary training laymen perform at the same level as skilled examiners. For genuine images, the average TNR is $88.47 \%$, and the unskilled examiners perform better $(88.81 \%)$ than skilled $(85.71 \%)$. Both TPR and TNR values are by far higher than in [7], indicating that digital images are significantly easier to classify than printed ones with the passport dimensions of $35 \times 45 \mathrm{~mm}$.

4.1.3 Results of algorithms. The detectors are tuned to deliver the optimal performance with the default threshold of 0.5 . The keypoint detector yields $100 \%$ TPR $=0 \%$ FNR (0/10 misses) and $80 \%$ TNR $=20 \%$ FPR ( $1 / 5$ false alarms). The minimal HTER of $10 \%$ can be obtained with the decision thresholds between 0.032 and 0.763 (see Figure 5a). The DCNN-based detector yields $80 \%$ TPR $=20 \% \mathrm{FNR}(2 / 10$ misses $)$ and $100 \%$ TNR $=0 \%$ FPR $(0 / 5$ false alarms). The minimal HTER of $0 \%$ can be obtained with the decision thresholds between 0.0000003 and 0.000627 (see Figure $5 b)$. Note that setting such a marginal threshold is not practical.

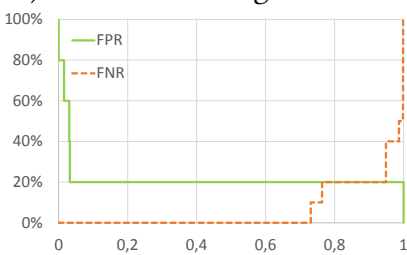

(a)

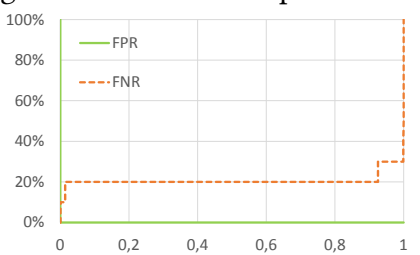

(b)
Figure 5: Error rates of MAD approaches: (a) keypoints, (b) DCNN

4.1.4 Humans vs. algorithms. Morphing detection performance of humans and algorithms are compared in Table 1. Even with default thresholds, MAD approaches outperform humans, which can be seen by comparing HTER values. Humans also detect on average fewer morphs than the MAD approaches, but only the DCNN-based detector has fewer false alarms than humans.

Table 1: Comparison of morphing detection rates of humans and MAD.

\begin{tabular}{llll}
\hline Approach & TPR & TNR & HTER \\
\hline \hline Skilled examiners & $63.33 \%(133 / 210)$ & $85.71 \%(90 / 105)$ & $25.48 \%$ \\
\hline Unskilled exam. & $65.65 \%(1103 / 1680)$ & $88.81 \%(746 / 840)$ & $22.77 \%$ \\
\hline All examiners & $65.40 \%(1236 / 1890)$ & $88.47 \%(836 / 945)$ & $23.07 \%$ \\
\hline Keypoints $(\tau=0.5)$ & $100 \%(10 / 10)$ & $80 \%(4 / 5)$ & $10 \%$ \\
\hline DCNN $(\tau=0.5)$ & $80 \%(8 / 10)$ & $100 \%(5 / 5)$ & $10 \%$ \\
\hline DCNN $(\tau=0.0006)$ & $100 \%(10 / 10)$ & $100 \%(5 / 5)$ & $0 \%$ \\
\hline
\end{tabular}

\subsection{Matching experiment}

4.2.1 Performance metrics. The metrics for matching performance are adopted from biometrics (false reject rate (FRR) and false accept rate (FAR)) and complemented by the morph accept rate(MAR).There is no need for mated metrics as proposed in[22] because morphs are checked against only one constituent face.

4.2.2 Results of humans. Figure $4 \mathrm{~b}$ shows the matching error rates of humans. The average MAR is $57.88 \%$. Surprisingly, skilled examiners perform worse than unskilled (average MAR
$61.90 \%$ vs. $57.38 \%$ ). The same tendency can be observed with FAR $(16.19 \%$ vs. $15.36 \%)$. In contrast, the FRR of skilled examiners is lower (21.90\% vs. $23.75 \%$ ). The average FAR of $15.45 \%$ and FRR of $23.54 \%$ are quite high, which corresponds to the results of matching unfamiliar faces in [2]. Note that the average MAR is notably higher than average FAR, characterizing morphs to be a serious threat for humans.

4.2.3 Results of algorithms. The error diagrams of the AFR systems are shown in Figure 6. With the predefined threshold $\tau=80$, Dermalog yields $0 \%$ FAR $(0 / 5), 0 \%$ FRR $(0 / 10)$, but $80 \%$ MAR $(8 / 10)$. Keeping FAR and FRR equal to $0 \%$, the minimal MAR of $30 \%$ can be obtained with the thresholds in the interval [75.57, 89.87] (see Figure 6a). The MAR and FRR of Dlib with the predefined threshold $\tau=0.6$ are equal to those of Dermalog with $\tau=80$, but the FAR rate yields $40 \%(2 / 5)$. With a threshold from the interval $[0.44,0.53]$, the FRR and FAR would yield $0 \%$. The minimal MAR of $30 \%$ would be obtained on the left margin of this interval $\tau=0.44$ (see Figure $6 b$ ).

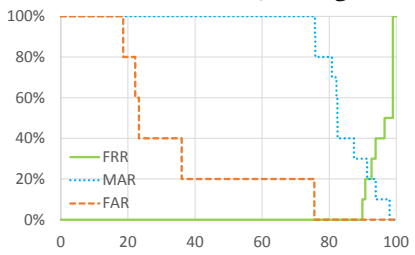

(a)

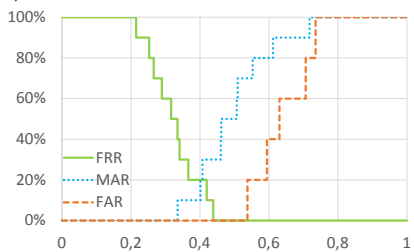

(b)
Figure 6: Error rates of AFR systems: (a) Dermalog, (b) Dlib

4.2.4 Humans vs. algorithms. Matching performances of humans and algorithms are compared in Table 2. Both AFR systems with the optimal threshold clearly outperform humans in all three aspects: accepting genuine trials (FRR), rejecting impostor trials (FAR), and rejecting morphing trials (MAR). However, with the standard thresholds, the MAR of both face matchers yields $80 \%$ which is higher than that of humans (57.88 $\%$ ). Dermalog with FAR and FRR of $0 \%$ outperforms humans while the FAR of Dlib is more than twice as high as of humans.

Table 2: Comparison of matching error rates of humans and AFR systems.

\begin{tabular}{llll}
\hline Approach & MAR & FAR & FRR \\
\hline \hline Skilled examiners & $61.90 \%(130 / 210)$ & $16.19 \%(17 / 105)$ & $21.90 \%(46 / 210)$ \\
\hline Unskilled exam. & $57.38 \%(964 / 1680)$ & $15.36 \%(129 / 840)$ & $23.75 \%(399 / 1680)$ \\
\hline All examiners & $57.88 \%(1094 / 1890)$ & $15.45 \%(146 / 945)$ & $23.54 \%(445 / 1890)$ \\
\hline Dermalog $(\tau=80)$ & $80 \%(8 / 10)$ & $0 \%(0 / 5)$ & $0 \%(0 / 10)$ \\
\hline Dermalog $(\tau=89.87)$ & $30 \%(3 / 10)$ & $0 \%(0 / 5)$ & $0 \%(0 / 10)$ \\
\hline Dlib $(\tau=0.6)$ & $80 \%(8 / 10)$ & $40 \%(2 / 5)$ & $0 \%(0 / 10)$ \\
\hline Dlib $(\tau=0.44)$ & $30 \%(3 / 10)$ & $0 \%(0 / 5)$ & $0 \%(0 / 10)$ \\
\hline
\end{tabular}

\subsection{Morphing indicators and decision time}

Here we analyze, focusing on which face region contributes the most to the correctness of human decisions. First, we separate correct and wrong decisions and repeat the following procedure for both groups. For each trial we count the proportion of decisions with a focus on a particular face region in the total number of decisions and count an average from all trials. The focus is a binary flag derived from an answer to the question "Which face parts did you mostly focus on?" with 1 for "almost always" and 0 for any other choice. The results are in Figure 7. A green bar can be interpreted as the likelihood of a correct decision if the focus 
of an examiner is on a particular region and an orange bar the likelihood of a wrong decision. The difference between two bars is of interest. Based on this, eyes are the most important region followed by nose and mouth. If the focus is on skin or face contour, correct or wrong decisions are almost equally frequent. If the focus is on hair, wrong decisions are more frequent. Note that this analysis is done only for the morphing detection part.

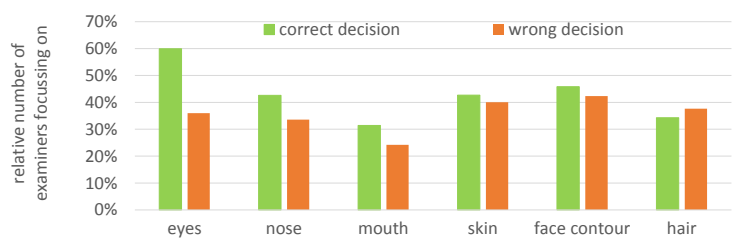

Figure 7: Face regions contributed the most to the correct and wrong decisions of humans in the detection experiment.

The error bars in Figure 8 visualize average decision time along with the mean absolute deviation for each trial. Matching is on average significantly slower than detection, $16.88 \mathrm{~s}$ vs. $10.31 \mathrm{~s}$ per sample. Surprisingly, skilled examiners are slower in both parts. For detection the time difference is rather low (11.32s vs. 9.31s) except for the sample M5 for which one skilled examiner took 13 minutes to decide. For matching the time difference is higher (22.55s vs. 18.15s). Variations in decision time for matching are dramatically higher than for detection. Moreover, decision time clearly correlates with the complexity of a sample (14s for M2 vs. 7s for M3 in the detection part and 30s for M1 vs. 14s for M10 in the matching part). In the matching part, impostor trials require on average notably less time than genuine or morphing trials. The likelihood to make a correct decision does not depend on the time taken and strongly varies from one examiner to another.
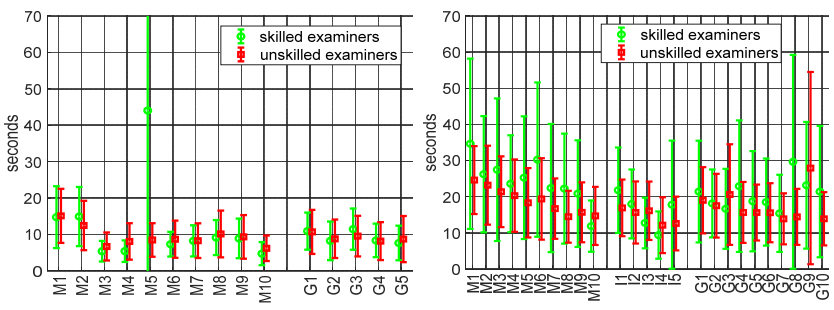

Figure 8: Decision time in sec. of skilled and unskilled examiners for the detection experiment (left) and matching experiment (right).

\section{Conclusion}

This paper introduces a revision of our ongoing web-based experiment simulating border control by examining humans to detect morphed face images and to match potentially morphed passport images against "live" faces of travelers. We observed the average TPR of $65.40 \%$ and TNR of $88.47 \%$ in the detection part and the average MAR of $57.88 \%$, FAR of $15.45 \%$, and FRR of $23.54 \%$ in the matching part. We discovered no significant performance difference for skilled und unskilled participants. Surprisingly, in many cases unskilled participants were even more successful than skilled. This can be explained by the usage of high-quality morphs and vanishing of morphing artifacts after re-digitalization. Skilled examiners tend to overthink and find artifacts where they do not exist. The performance of humans in both parts is very poor. In contrast, two MAD approaches and two AFR systems with properly set decision thresholds yield far lower error rates. Nonetheless, even for this small sample with only 15 detection and 25 matching trials, none of the algorithms is able to perform without errors with a predefined threshold and the MAR of both AFR systems are dramatically high. Hence, we conclude that computer-aided support of human examiners is required, but the considered algorithms are not reliable enough to replace border officers. The better performance in the detection part suggests increasing an MRTD chip memory to store digital images of higher resolution and avoiding re-digitalization.

\section{ACKNOWLEDGMENTS}

This work has been funded in part by the German Federal Ministry of Education and Research (BMBF) through the research program ANANAS under the contract no. 16KIS0509K. We thank our students Lukas Meißner, Oliver Schade, Tobias Schmidt and Lena Zess for the implementation of the experiment We also thank all models and examiners of their contributions.

\section{REFERENCES}

[1] P. Hancock, V. Bruce, A.M. Burton, 2000. Recognition of unfamiliar faces. Trends in Cog. Sci. 4(9), 330-337.

[2] D. White, R.I. Kemp, R. Jenkins, M. Matheson, A.M. Burton, 2014. Passport Officers' Errors in Face Matching. PLoS ONE 9(8), e103510.

[3] R. Raghavendra, C.Busch, 2017. Presentation Attack Detection Methods for Face Recognition Systems: A Comprehensive Survey, ACM Comp. Surv. 50(1), 8

[4] M. Ferrara, A. Franco, D. Maltoni, 2014. The Magic Passport, In Proc. IEEE Int. foint Conf. on Biometrics, 1-7.

[5] M. Ferrara, A. Franco, D. Maltoni, 2016. On the Effects of Image Alterations on Face Recognition Accuracy. In Bourlai, T. (ed.) Face Recognition Across the Electromagnetic Spectrum, Springer, 195-222.

[6] U. Scherhag, R. Raghavendra, K.B. Raja, M. Gomez-Barrero, C. Rathgeb, C. Busch, 2017. On the Vulnerability of Face Recognition Systems: Towards Morphed Face Attacks, In Proc IWBF'17, 1-6.

[7] A. Makrushin, T. Neubert, J. Dittmann, 2017. Automatic generation and detection of visually faultless facial morphs, In Proc. VISAPP'17, 39-50.

[8] A. Makrushin, T. Neubert, J. Dittmann, 2019. Humans Vs. Algorithms: Assessment of Security Risks Posed by Facial Morphing to Identity Verification at Border Control, In Proc. VISAPP'19, 513-520.

[9] P.J. Phillips, A.J. O’Toole, 2014. Comparison of human and computer performance across face recognition experiments. Image\&Vis.Comp. 32, 74-85.

[10] D.J. Robertson et al., 2017. Fraudulent ID using face morphs: Experiments on human and automatic recognition, PLoS ONE 12(3), e0173319.

[11] D.J. Robertson et al., 2018. Detecting morphed passport photos: a training and individual differences approach. Cog. Res.: Principles and Impl. 3, 27.

[12] R.S.S. Kramer et al., 2019. Face morphing attacks: Investigating detection with humans and computers, Cognitive Research: Principles and Implications 4, 28.

[13] T. Neubert, A. Makrushin, M. Hildebrandt, C. Kraetzer, J. Dittmann, 2018. Extended StirTrace Benchmarking of Biometric and Forensic Qualities of Morphed Face Images, IET Biometrics 7(4), 325-332.

[14] C. Kraetzer et al., 2017. Modeling Attacks on Photo-ID Documents and Applying Media Forensics for the Detection of Facial Morphing, In Proc. IH\&MMSec '17, 21-32.

[15] C. Seibold, W. Samek, A. Hilsmann, P. Eisert, 2018. Accurate and Robust Neural Networks for Security Related Applications Exampled by Face Morphing Attacks. CoRR abs/1806.04265.

[16] C. Seibold, W. Samek, A. Hilsmann, P. Eisert, 2017 Detection of Face Morphing Attacks by Deep Learning. In Proc. IWDW'17, 107-120.

[17] International Civil Aviation Organization, 2018. Portrait Quality (Reference Facial Images for MRTD), Draft Technical Report, Version 1.0.

[18] DERMALOG Identification Systems $\mathrm{GmbH}$, Face Recognition Software, https://www.dermalog.com/products/software/face-recognition/

[19] D. King, 2009. Dlib-ml: a machine learning toolkit. F. Mach. Learn. Res. 10, 17551758.

[20] D. King, Dlib C++ Library, Release notes, http://dlib.net/release_notes.html, last accessed 26.04.2020

[21] ISO/IEC JTC1 SC37 Biometrics, ISO/IEC 30107-3:2017 Information technologyBiometric presentation attack detection - Part3: Testing \& reporting

[22] U. Scherhag et al., 2017. Biometric Systems under Morphing Attacks: Assessment of Morphing Techniques and Vulnerability Reporting," In Proc. BIOSIG'17, 149-159. 